\title{
Study of Backfire Antennas
}

\author{
G. S. Kirov ${ }^{*}$, H. D. Hristov** \\ *Department of Radio Engineering, Technical Uniersity of Varna, Studentska Str.1, 9010 Varna, Bulgaria \\ e-mail: gkirov@abv.bg \\ **Departamento de Electrónica, Universidad Técnica Federico Santa Maria, Valparaiso, Chile \\ e mail: h.hristov@ieee.org
}

\begin{abstract}
The study presented in this article is dedicated to the backfire disk-on-rod antennas fed by a dipole or crossed dipole. For short, these are herein called backfire antennas. Backfire antenna constructions with different configurations of the big reflector are considered. The influence of the feed location in the backfire antenna construction upon the antenna characteristics is also examined. The studies were carried out with a corrugated-rod (disks attached to a metal rod) surface-wave structure. The results obtained may be applied to other types of surface-wave structures, such as dipole array, dielectric rod or dielectric-covered metal rod. The lengths of the investigated antennas ranged between $2 \lambda$ and $4 \lambda$. The design carried out in these investigations may be used for creation of high efficiency backfire antennas.
\end{abstract}

Index Terms - backfire antenna, end-fire antenna, long backfire antenna, surface wave, surface-wave antenna, surface-wave structure.

\section{INTRODUCTION}

The backfire antenna is created by H. W. Ehrenspeck and his associates at the Air Force Cambridge Research Center, Bedford, Mass. in 1959 [1]-[5]. It was obtained by placing of a big reflector at the open end of an end-fire antenna perpendicularly to its axis. Thus, the antenna increases its length for the surface wave which leads to the improvement of the antenna gain and directivity.

The geometry of the backfire antenna is shown in Fig. 1. It consists of a source $F$ (for example, a dipole or crossed dipole), a surface-wave structure $S$ (dipole array, corrugated rod, dielectric rod or dielectric-covered metal rod) and two parallel disk reflectors: small reflector $R_{1}$ and big reflector $R_{2}$. In the figure, the antenna length is the distance between the reflectors $R_{1}$ and $R_{2}$, denoted by $L$. The radiation mechanism of the backfire antenna is described in many references [4]-[10]. The first, simplified model of the backfire antenna radiation mechanism was very rough approximation to the real physical process in the antenna. It presents the backfire antenna as an end-fire antenna of effective length $L_{e}$ equal to double the physical length $L$, or $L_{e}=2 L$. The spherical wave radiated from the source $F$ is transformed in a surface wave $S W_{1}$ by the surface-wave structure $S$. It terminates by the big reflector $R_{2}$, which reflects the surface wave $S W_{2}$ toward the small reflector $R_{1}$, where it is radiated from the antenna aperture $V V^{\prime}$ into the space. Thus, the radiation of the antenna is directed in inverse direction in comparison with the radiation of the ordinary end-fire antenna used as a backfire antenna prototype. Because of this reason it is called backfire antenna (antenne à rayonnement inverse, антенна обратного излучения, etc...). According to this model the directivity increase of 
the backfire antenna in comparison with the end-fire antenna should be 4 times (or $6 \mathrm{~dB}$ ): $3 \mathrm{~dB}$ due to the length of the antenna $L_{e}=2 L$ (the directivity of the relatively short surface-wave antenna is proportional to its length) and $3 \mathrm{~dB}$ due to the presence of the big reflector which assures a radiation from the antenna in only one hemisphere.

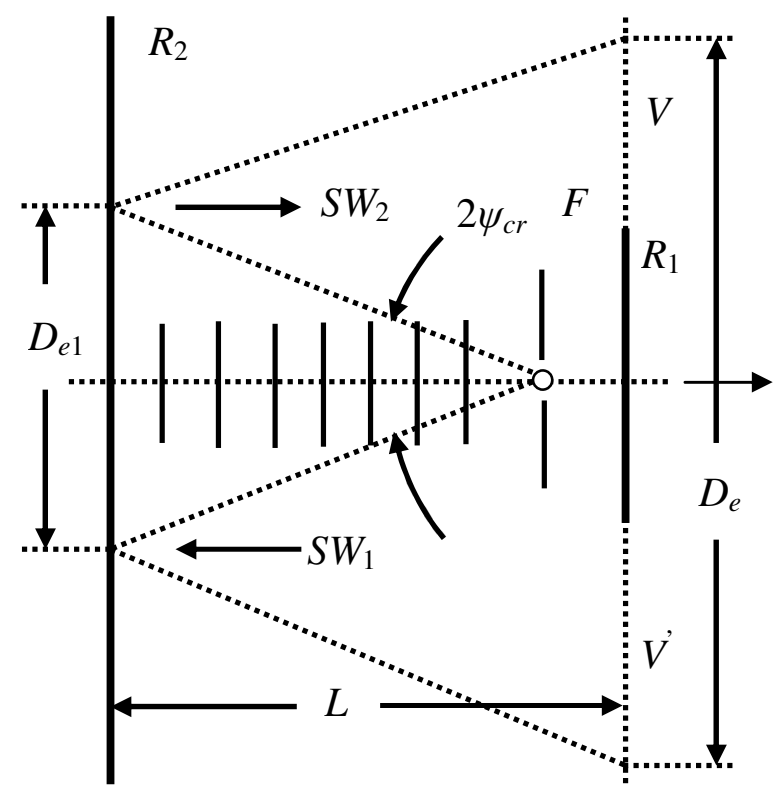

Fig. 1. The geometry of a backfire antenna.

It is found in practice that a bigger directivity increase (6 times or approximately $8 \mathrm{~dB}$ ) may be obtained by the backfire antenna using a phase corrected big reflector and accurate tuning of the surface-wave structure. This result may be explained only by means of more rigorous model of the backfire antenna radiation mechanism. Consistent with this model the surface wave is reflected multiple times between the reflectors $R_{1}$ and $R_{2}$ of the semitransparent open cavity $R_{1} R_{2}$ before be radiated from the virtual antenna aperture $V V^{\prime}$. The diffraction effects on the both reflectors and the direct radiation from the feed dipole should be also taken into account [7]-[10]. Our experiments with backfire antennas have shown that $L_{e}$ equal to $6 L$ gives the best result in regard to the optimum value of the phase velocity of the surface wave. This model corresponds to six forward and backward travels of the surface wave along the surface-wave structure or to a triple wave reflection from the big reflector.

Because of its uncontestable advantages (simple and compact construction, reduced length and good radiation performance) the backfire antennas have been widely used in various wireless systems, mostly military, earth and spacecraft. Now they may be used for long-range point-to-point applications, say WLAN, WISP and satellite links and in those cases where a gain between $15 \mathrm{~dB}$ and $25 \mathrm{~dB}$ and a bandwidth less than $10 \%$ are required.

\section{OPTIMUM PHASE VELOCITY AND DIMENSIONS OF THE BACKFIRE ANTENNA}

In order to obtain maximum directivity and efficiency from the backfire antenna the phase velocity 
of the surface wave and the dimensions of the antenna should have optimum values.

\section{A. Phase velocity}

According to the Hansen-Woodyard condition [11], [12] the optimum value of the phase velocity delay factor of an uniformly illuminated end-fire antenna is

$$
c / v=1+\lambda_{0} /(2 L), L>20 \lambda_{0}
$$

where $c$ is the free-space wave velocity, $v$ is the phase velocity of the surface wave, and $\lambda_{0}$ corresponds to $f_{0}-$ the design (central or resonant) frequency.

The directivity of the end-fire antenna above an isotropic radiator in these conditions is

$$
D=7 L / \lambda_{0}
$$

If the design is based on the optimum phase velocity and taper dimensions of the surface-wave structure (non-uniformly illumination) the expressions (1) and (2) become

$$
\begin{gathered}
c / v=1+\lambda_{0} /(q L), \\
D=10 L / \lambda_{0}, 3 \lambda_{0}<L<8 \lambda_{0},
\end{gathered}
$$

where the coefficient $q$ starts near 6 for $L=\lambda_{0}$ and diminishes to approximately 3 for $L$ between $3 \lambda_{0}$ and $8 \lambda_{0}$ and to 2 at $20 \lambda_{0}$. The formulas (3) and (4) proposed by Ehrenspeck and Poehler [12], [13] are based on the experimental investigations on Yagi-Uda antennas.

According to the Hansen-Woodyard condition and based on the simplified model of the backfire antenna radiation mechanism [4], [6], [14] the optimum value of the phase velocity delay factor and the directivity are given by

$$
\begin{gathered}
c / v=1+0.234\left(\lambda_{0} / L\right), L>10 \lambda_{0}, \\
D=28 L / \lambda_{0}
\end{gathered}
$$

The change of the phase velocity delay factor $c / v$ as a function of the backfire antenna length in wavelengths $L / \lambda_{0}$ is shown in Fig. 2(a) with a dotted line. Better results can be obtained using Ehrenspeck and Poehler experimental results [4]:

$$
\begin{gathered}
c / v=1+\lambda_{0 /}(2 q L), \\
D=40 L / \lambda_{0}, 1.5 \lambda_{0}<L<4 \lambda_{0}
\end{gathered}
$$

The variation of the phase velocity delay factor $c / v$ consistent with the expression (7) is presented in above figure with a dashed line. Finally, consistent with the model of the backfire antenna radiation mechanism proposed herein $\left(L_{e}=3 L\right)$ the optimum value of $c / v$ (Fig. 2(a),solid line) and the directivity can be defined as follows

$$
\begin{gathered}
c / v=1+0.078 \lambda_{0} / L, \\
D=60 L / \lambda_{0}, 1.5 \lambda_{0}<L<4 \lambda_{0}
\end{gathered}
$$




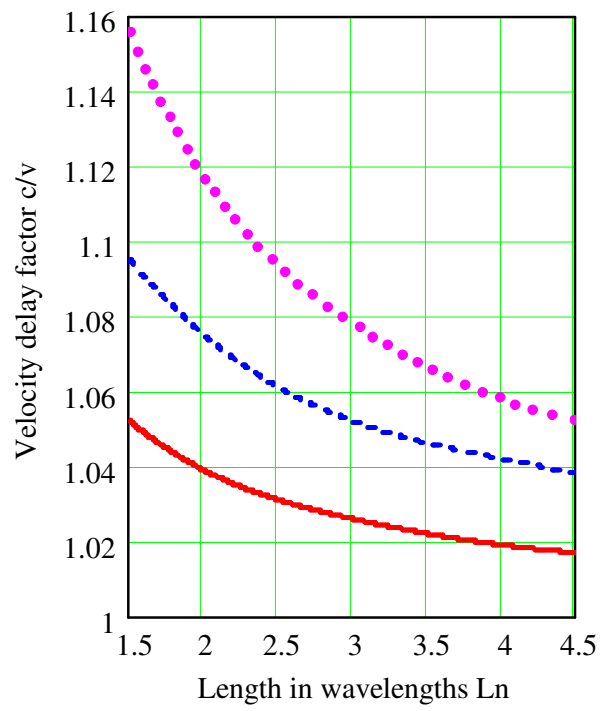

(a)

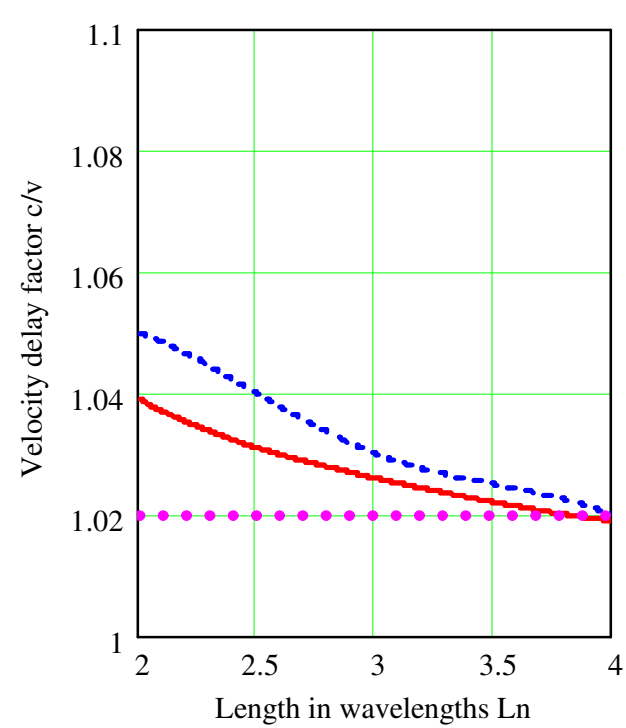

(b)

Fig. 2. Phase velocity delay factor versus backfire antenna length in wavelengths $L n=L / \lambda_{0}$ : (a) different models of radiation mechanism: solid line: Hristov and Kirov model - eq. (9); dashed line: Ehrenspeck and Poehler experimental values eq. (7); dotted line: Hansen - Woodyard condition - eq. (5); (b) comparison between theory and measurements: solid line: Hristov and Kirov model - eq. (9); dashed line: Strom experimental values [5]; dotted line: Hristov and Kirov experimental values.

In order to verify the validity of the proposed model of radiation mechanism the both curves of the phase velocity delay factor, the solid line according to the proposed model and the dashed line consistent with the experimental results obtained by Strom [5] are presented in Fig. 2(b). In the same figure our experimental results for two types of backfire antennas: an antenna with a feed near the big reflector (dashed line coinciding with the Strom's results) and an antenna with a feed near the small reflector (dotted line) are also shown. A good agreement between the proposed model and the measurement is found.

\section{B. Feed location}

The feed located between the two reflectors acts directly on the backfire antenna characteristics. There are only two feed locations which assure normal work of the antenna. In the first manner of feed the feed dipole $F$ is located near the small reflector $R_{1}$. The backfire antenna with this feed location is preferable in the practice because of its good radiation characteristics. In the second manner of feed the feed dipole $F$ is located near the big reflector $R_{2}$. The use of the backfire antenna with this feed location is limited because of its lower gain (with 3-4 dB) compared to the antenna with the first feed location. In the both cases the distance between the feed point and respective reflector is $\lambda_{0} / 4$. Other points of feed location along the length of the backfire antenna are not suitable and are not recommended [10].

\section{Big reflector}

The diameter of the big reflector $R_{2}$ is related to the quantity of the reflected energy and to the phases of the reflected waves. In fact, the big reflector reflects two types of waves: a surface wave 
$S W_{1}$ with phase velocity $v$ and a spherical wave with velocity $c$. In the plane of the big reflector, in the limits of so-called critical angle $2 \psi_{c r}$ (or $2 \psi<2 \psi_{c r}$ ), the wave has a quasi-plane phase front and effective radiation aperture of diameter $D_{e 1}$ (Fig. 1):

$$
D_{e 1}=2 L \tan \psi_{c r},
$$

where

$$
\tan \psi_{c r}=\sqrt{(c / v)^{2}-1}
$$

Having in mind (5) and (12) the diameter $D_{e 1}$ is defined as a function of $L / \lambda_{0}$ as follows

$$
D_{e 1}=1.4 \sqrt{L / \lambda_{0}}
$$

The value $2 \psi_{c r}$ divides the big reflector $R_{2}$ into two regions. In the first region with $2 \psi<2 \psi_{c r}$ the surface wave is predominant, while in the second one with $2 \psi>2 \psi_{c r}$ the spherical wave plays essential role in the wave distribution. Thus, the minimum diameter $D_{2}$ of the backfire antenna big reflector $R_{2}$ should be equal to the constant-phase effective aperture, or $D_{2}=D_{\mathrm{e} 1}$. If the diameter $D_{2}$ increases (the second region with $2 \psi>2 \psi_{c r}$ ) the phase front becomes spherical and a quadratic phase distortion on the big reflector plane occurs. In the case where a tolerable phase distortion of $\pi / 2 \mathrm{rad}$ at the disk edge is assumed, the dimension of its diameter is given by [4]

$$
D_{2}=2 \lambda_{0} \sqrt{L / \lambda_{0}}
$$

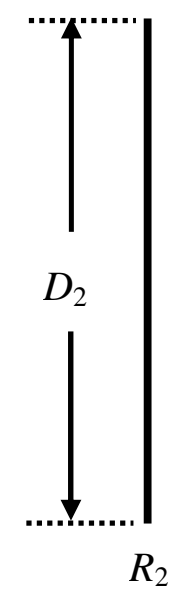

(a)

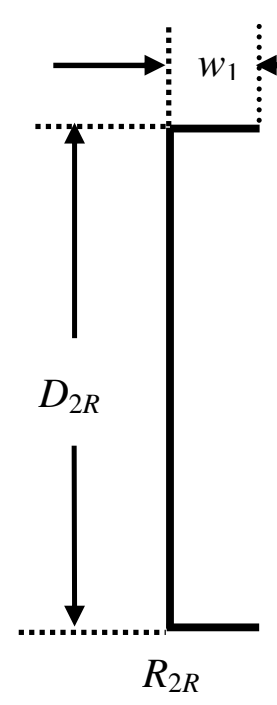

(b)

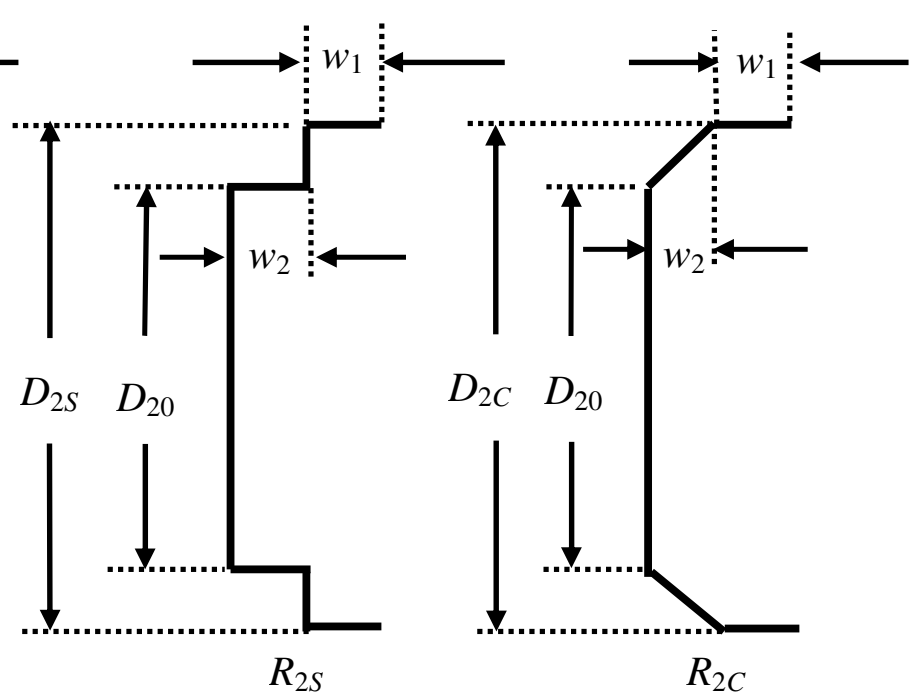

(c)

(d)

Fig. 3. Some possible configurations of the big reflector: (a) disk reflector $R_{2}$, (b) rimmed disk reflector $R_{2 R}$, (c) rimmed reflector with step correction $R_{2 S}$, and (d) rimmed reflector with conical correction $R_{2 C}$.

Some possible configurations of the big reflector are shown in Fig. 3. Fig. 3(a) shows a plane circular reflector $R_{2}$. Its diameter $D_{2}$ is defined by the expression (14). A rimmed disk reflector $R_{2 R}$ of 
diameter $D_{2 R}$ is shown in Fig. 3(b). The rim decreases the levels of the backlobe and sidelobes and leads to an improvement of the antenna gain and directivity. The diameter $D_{2 R}$ is calculated by the expression (14) and the rim width $w_{1}$ is equal to a quarter-wave length

$$
w_{1}=0.25 \lambda_{0}
$$

Further increase of the antenna directivity and efficiency can be obtained using a phase correction in big reflector $R_{2}$. Thus, the reflected wave will involve and the energy of the peripheral spherical waves with a minimum phase shift [4],[10]. In this manner the ideal big reflector should consist of two parts: a first plane part and a second parabolic one. In practice because of technological reasons the simplified stepped and conically corrected big reflectors are mostly used. Fig. 3(c) shows a rimmed stepped-corrected disk reflector $R_{2 S}$ of diameter $D_{2 S}$ with a inner diameter $D_{20}$ and step width $w_{2}$. A rimmed conically-corrected disk reflector $R_{2 C}$ of diameter $D_{2 C}$ is shown in Fig. 3(d). The corresponding dimensions of the reflectors $R_{2 S}$ and $R_{2 C}$ are given by the expressions [4],[10]

$$
\begin{gathered}
D_{20}=(1.9 \div 2.0) \lambda_{0} \sqrt{L / \lambda_{0}}, \\
D_{2 S}=D_{2 C}=(2.9 \div 3.0) \lambda_{0} \sqrt{L / \lambda_{0}}, \\
w_{1}=w_{2}=0.25 \lambda_{0},
\end{gathered}
$$

which are in good agreement with the results of the experimental studies.

\section{Length of the antenna}

The backfire antenna can be considered as a parallel-plane resonator antenna with a standing surface-wave field excited inside. In the virtual antenna aperture $V V^{\prime}$ (Fig. 1) two basic phenomena take place: multiple partial reflections from the small reflector $R_{1}$ and multiple intensive radiations from the open aperture area. The length of such radiating cavity must satisfy equation

$$
L=n \lambda_{s 0} / 2+\Delta L, \text { for } n=1,2,3, \ldots
$$

where $\lambda_{S 0}=v / f_{0}$ is the surface wave wavelength, $\Delta L$ is the length correction due to the radiation, and $n$ is a real integer called standing-wave number. The experimental results show that

$$
L=n\left(\lambda_{0} / 2\right)
$$

where $n$ have the same integral values as in (19).

On the other hand, the antenna length depends directly on the required directivity (gain). It can be approximately calculated by the expression

$$
L \approx(D / A) \lambda_{0}
$$

where the values of the coefficient $A$ are given in a Table I.

The accurate length of the backfire antenna can be defined by the mutual solution of equations (20) and (21). 
TABLE I. THE VALUES OF THE COEFFICIENT $A$ FOR A BACKFIRE ANTENNA, $L=2 \Lambda_{0}$ TO $4 \Lambda_{0}$

\begin{tabular}{|l|c|}
\hline \multicolumn{1}{|c|}{ Type of the big reflector } & Coefficient $\boldsymbol{A}$ \\
\hline $\mathrm{R}_{2 \mathrm{R}}$, a feed near the big reflector & $20-25$ \\
\hline $\mathrm{R}_{2 \mathrm{R}}$, a feed near the small reflector & $40-50$ \\
\hline $\mathrm{R}_{2 \mathrm{C}}$, a feed near the small reflector & 60 \\
\hline
\end{tabular}

The most often used in practice are the backfire antennas with lengths between $2 \lambda_{0}$ and $6 \lambda_{0}$. If a higher directivity is required an use of backfire antenna arrays is recommended.

\section{E. Small reflector}

The dimension of the small reflector $R_{1}$ acts essentially on the amplitude and phase field distribution in the antenna aperture. In addition it is related to the feed efficiency in the case where the feed is set near the small reflector. The very small values of this reflector transform the backfire antenna into an ordinary end-fire antenna and decrease the directivity. On the other hand the high values of the small reflector diameter close to the big reflector diameter also decrease the antenna directivity because of a reduction of the aperture area (the antenna transforms in a high quality-factor resonator).

The optimum value of the small reflector diameter increases with an increase of the antenna length and can be found by [10]

$$
D_{1}=(0.25 \div 0.45) \lambda_{0} \sqrt{L / \lambda_{0}}
$$

The experimental studies show that for a backfire antenna with a length between $2 \lambda_{0}$ and $6 \lambda_{0}$ the optimum value of the small reflector diameter ranges between $0.35 \lambda$ and $1.1 \lambda_{0}$.

\section{DESIGN PROCEDURE FOR BACKFIRE ANTENNAS}

There are only a few references dedicated to the backfire antennas design [4],[10]. It is not considered even in some recently published books on an antenna design [12],[15]-[17]. A design procedure for backfire antennas with circular reflectors and a corrugated-rod surface-wave structure, fed by a dipole, is described here. It is based on studies in [10], and some new results obtained by the authors. The results presented here may be applied to other types of surface-wave structures, such as dipole array, dielectric rod, or dielectric-covered metal rod. The lengths of the antennas studied ranged between $2 \lambda_{0}$ and $4 \lambda_{0}$, which are the most-used lengths, in practice. However, the range of the proposed design may be extended by a simple extrapolation in the range of the antenna lengths from $2 \lambda_{0}$ to $6 \lambda_{0}$.

Three constructions of backfire antennas are considered in this design:

- a backfire antenna fed by a dipole located near the big reflector $R_{2 R}$, an antenna A1 (Fig. 4);

- a backfire antenna fed by a dipole set near the small reflector with a big reflector $R_{2 R}$, an antenna A2 (Fig. 5); 


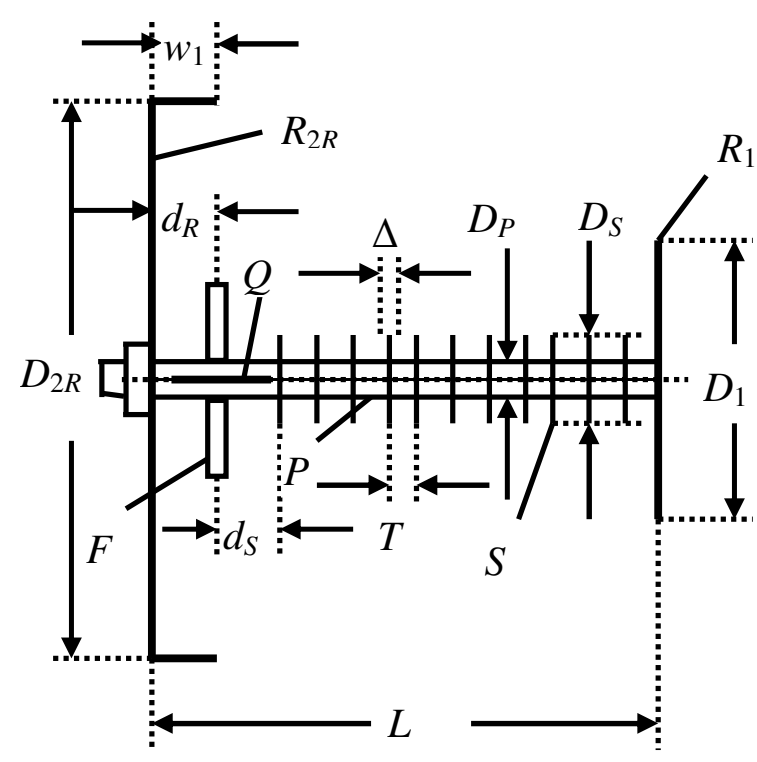

Fig. 4. A backfire antenna fed by a dipole located near the big reflector $R_{2 R}$ (Antenna $\mathrm{A} 1$ ).

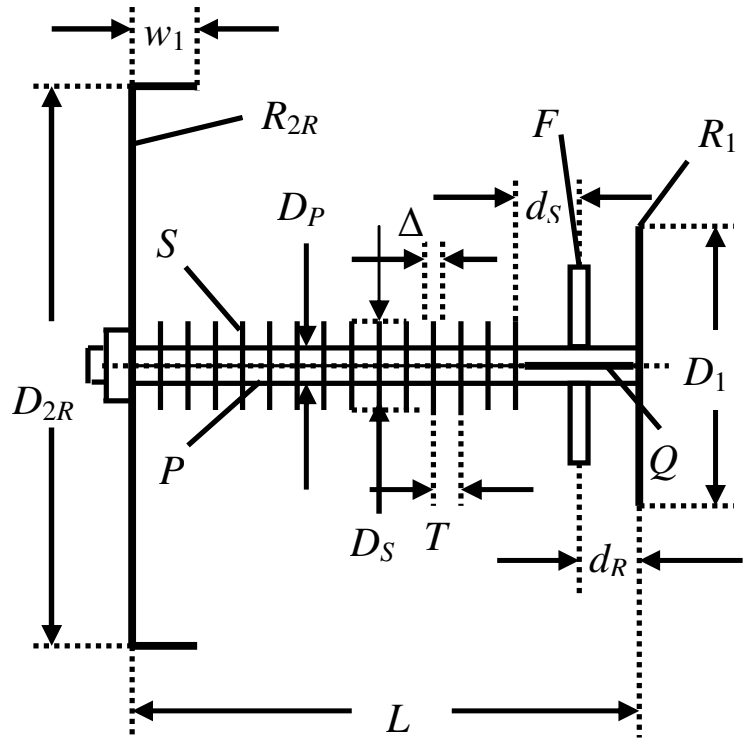

Fig. 5. A backfire antenna fed by a dipole set near the small reflector with a big reflector $R_{2 R}$ (Antenna A2).

- a backfire antenna fed by a dipole located near the small reflector with a big reflector $R_{2 C}$, an antenna A3 (Fig. 6).

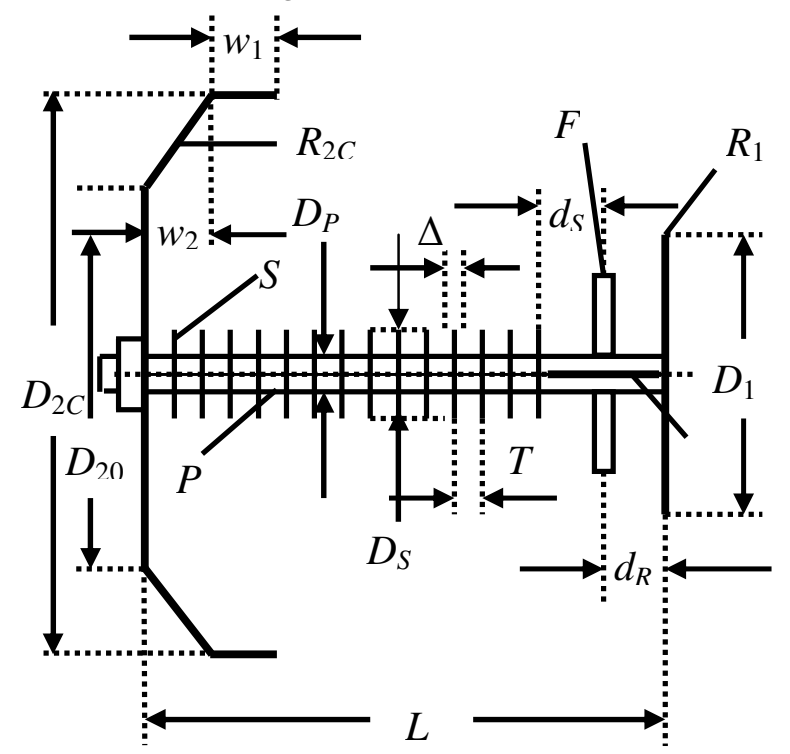

Fig. 6. A backfire antenna fed by a dipole located near the small reflector with a big reflector $R_{2 C}$

(Antenna A3).

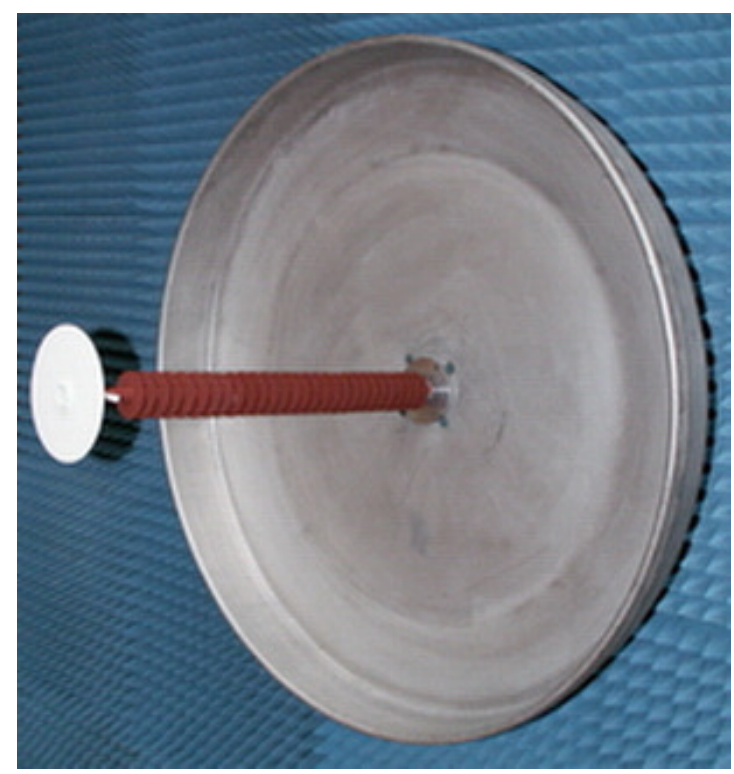

Fig. 7. The practical implementation of a backfire antenna A3, $L=4 \lambda_{0}$.

The antenna A1 has the most simple construction but its gain is the lowest while the antenna A3 provides the highest gain with relatively more complex construction. The new antenna elements and dimensions in Fig. 4 - Fig. 6 are denoted as follows: $P$ is a metal rod (a rigid feed coaxial line), $Q-$ a half-wavelength slot balun used to feed the symmetrical dipole by the coaxial line, $D_{1}-$ a small reflector diameter, $D_{P}-$ a metal rod diameter, $D_{S}-$ a disks diameter, $d_{S}$ and $d_{R}-$ distances between the dipole and the surface-wave structure, and the nearer reflector, respectively, $T-$ a period of the surface-wave structure, and $\Delta-$ a disks thickness. 
The directivity, $D$, the central frequency of the antenna's bandwidth, $f_{0}$, and the type of the surfacewave structure, $S$, are assumed known in this design. The design goal is to determine the basic dimensions of the backfire antenna. The design procedure involves the following steps:

\section{A. Length of the backfire antenna}

The approximate length of the antenna is defined by the required directivity

$$
L=\frac{10^{\frac{D[d B]}{10}}}{A} \lambda_{0}
$$

using the Table I for the values of the coefficient $A$.

The final length of the antenna is defined according to the requirements of (20) and (21).

\section{B. Optimum phase velocity of the backfire antenna}

The optimum value of the phase velocity delay factor $c / v$ is calculated by the expression (9). For backfire antennas with lengths between $2 \lambda_{0}$ and $4 \lambda_{0}$ more accurate values obtained experimentally by the authors are given in Table II.

TABLE II. THE OPTIMUM VALUES OF THE PHASE VELOCITY DELAY FACTOR C/V FOR A BACKFIRE ANTENNA FOR $L=2 \Lambda_{0}$ TO $4 \Lambda_{0}$

\begin{tabular}{|l|c|}
\hline \multicolumn{1}{|c|}{ Type of the backfire antenna } & Phase velocity delay factor $\boldsymbol{c} / \boldsymbol{v}$ \\
\hline Antenna 1 (Fig. 4) & \\
$L=2 \lambda_{0}$ & 1.05 \\
$L=3 \lambda_{0}$ & 1.03 \\
$L=4 \lambda_{0}$ & 1.02 \\
\hline Antenna 2 (Fig. 5) & 1.02 \\
\hline Antenna 3 (Fig. 6) & 1.02 \\
\hline
\end{tabular}

\section{Dimensions of the big reflector}

The diameter $D_{2 R}$ for the backfire antennas A1 and A2 is defined by formula (14) and the rim width $w_{1}$ is given by (15).

The diameter of the plane part of the big reflector $D_{20}$ for the backfire antenna A3 is calculated by (16) and the big reflector diameter $D_{2 C}$ is given by (17). The rim width $w_{1}$ and the step width $w_{2}$ are defined by (18).

\section{Diameter of the small reflector}

The optimum values of the small reflector diameter (obtained experimentally by the authors) are given in Table III.

TABLE III. THE DIAMETER OF THE SMALL REFLECTOR OF A BACKFIRE ANTENNA, FOR $L=2 \Lambda_{0}$ TO $4 \Lambda_{0}$

\begin{tabular}{|l|c|}
\hline \multicolumn{1}{|c|}{ Type of the backfire antenna } & Diameter $\boldsymbol{D}_{\mathbf{1}}$ \\
\hline Antenna 1 (Fig. 4) & $0.295 D_{2 R}$ \\
\hline Antenna 2 (Fig. 5) & $0.170 D_{2 R}$ \\
\hline Antenna 3 (Fig. 6) & $0.150 D_{2 C}$ \\
\hline
\end{tabular}

E. Distances from the dipole to the surface-wave structure, and the respective reflector

The both the distance $d_{S}$ and the distance $d_{R}$ are defined by 


$$
d_{S}=d_{R}=0.25 \lambda_{0}
$$

\section{F. Surface-wave structure}

Based on the optimum value of the ratio $c / v$ found above the surface-wave structure dimensions $D_{S}$, $D_{P}, T$ and $\Delta$ may be defined using the well known design procedure described in [18] or [16]. In the case where a dielectric rod is used as a surface-wave structure the design procedure should be accomplished consistent with the methods described for example in [16] or [12], [17].

\section{G. Feed dipole}

The design of the half-wavelength dipole (or crossed dipole) is classic and may be found in many references [12], [15]-[17].

\section{PRACTICAL EXAMPLES}

In order to verify the validity of the proposed design procedure, three backfire antennas were designed, fabricated and tested. A photograph of a backfire antenna with a corrugated-rod surface-wave structure fed by a dipole set near the small reflector with a big reflector $R_{2 C}$ and length $L=4 \lambda_{0}, \lambda_{0}=$ $13.6 \mathrm{~cm}$, is shown in Fig. 7. This antenna construction (an antenna A3) was chosen as a basic antenna configuration because of its high performance characteristics.

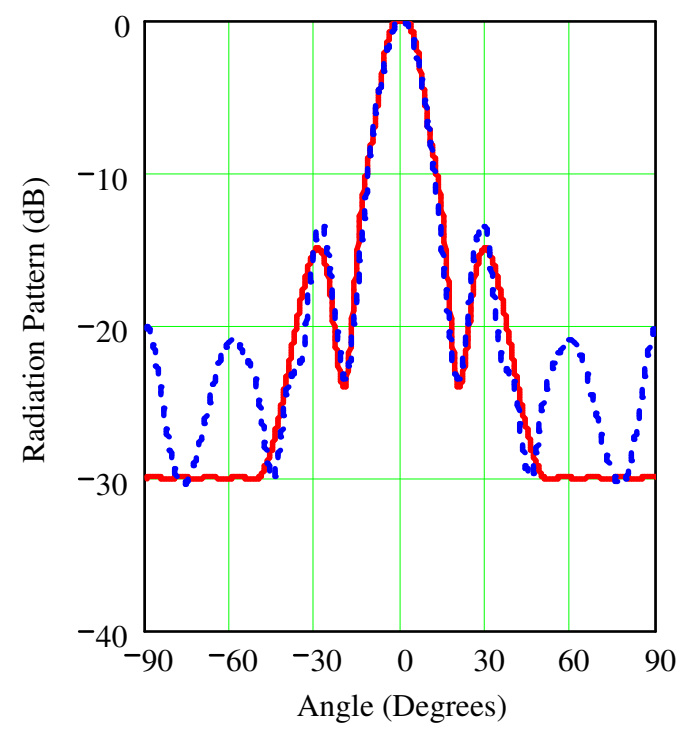

Fig. 8. The radiation patterns of a backfire antenna $\mathrm{A} 3$ in $E$-plane (solid line) and $H$-plane (dashed line): $L=2 \lambda_{0}, f=2.2 \mathrm{GHz}$.

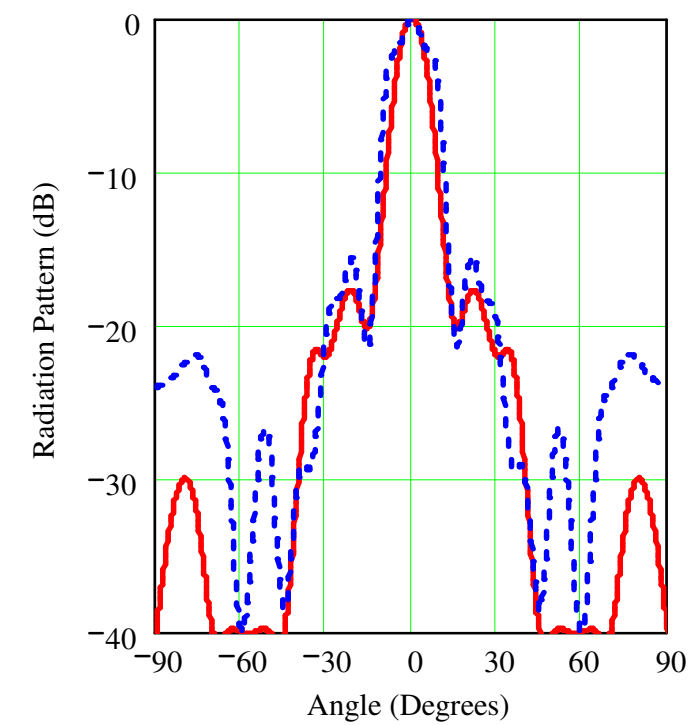

Fig. 9. The radiation patterns of a backfire antenna $\mathrm{A} 3$ in $E$-plane (solid line) and $H$-plane (dashed line): $L=3 \lambda_{0}, f=2.25 \mathrm{GHz}$.

The radiation patterns in $E$ - (solid line) and $H$-plane (dashed line) of the fabricated antennas with lengths $L=2 \lambda_{0}, 3 \lambda_{0}$, and $4 \lambda_{0}$ were measured and shown in Fig. 8 - Fig. 10, respectively. The directivities of the antennas were defined by an integration of their radiation patterns and the results obtained are illustrated in Fig. 11 for lengths $L=2 \lambda_{0}, f_{0}=2.2 \mathrm{GHz}$ (dotted line), $L=3 \lambda_{0}, f_{0}=2.25$ $\mathrm{GHz}$ (dashed line), and $L=4 \lambda_{0}, f_{0}=2.2 \mathrm{GHz}$ (solid line). Fig. 12 illustrates the input match bandwidth of the backfire antenna A3 with length $L=4 \lambda_{0}, \lambda_{0}=13.6 \mathrm{~cm}$. The bandwidth for a 2:1 
VSWR is $12 \%$ and for a $1.5: 1 \mathrm{VSWR}$ is $8.6 \%$, respectively. The influence of the antenna length on the bandwidth is insignificant.

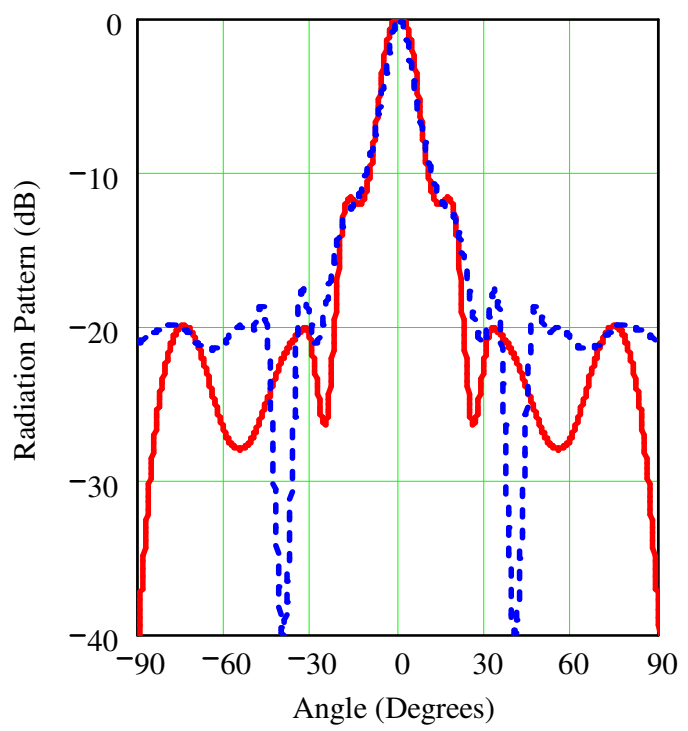

Fig. 10. The radiation patterns of a backfire antenna A3 in $E$-plane (solid line) and $H$-plane (dashed line): $L=4 \lambda_{0}$, $f=2.2 \mathrm{GHz}$.

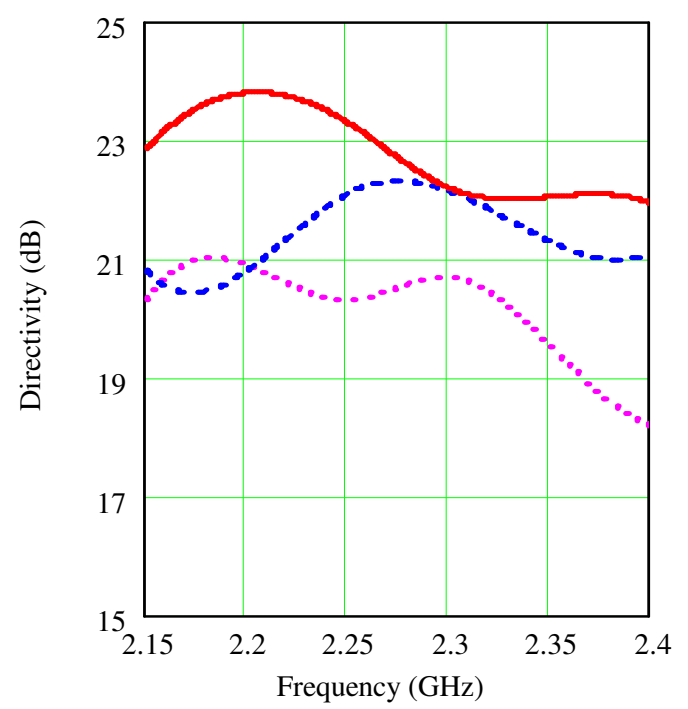

Fig. 11. The directivities of three backfire antennas A3 with lengths $L=2 \lambda_{0}$ (dotted line), $L=3 \lambda_{0}$ (dashed line), and $L=4 \lambda_{0}$ solid line).

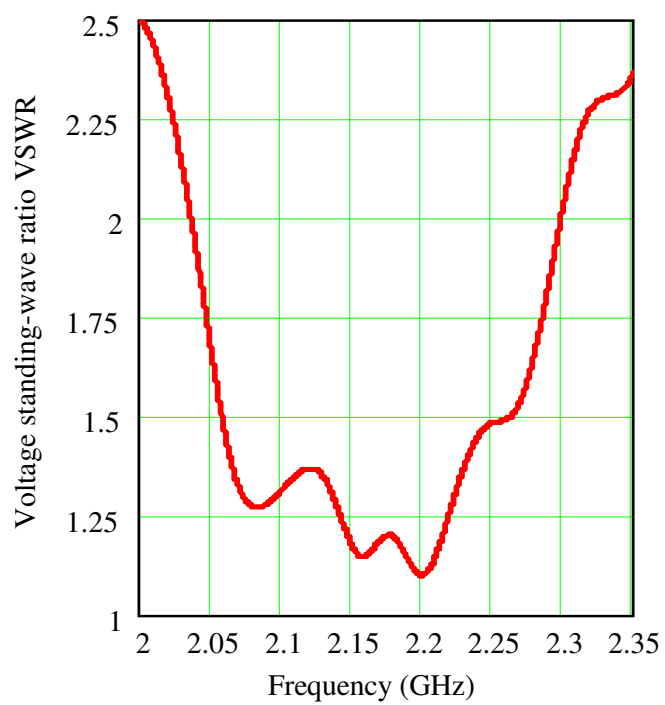

Fig. 12. Measured VSWR as function of frequency for a backfire antenna A3 with length $L=4 \lambda_{0}$.

The dimensions of the backfire antennas designed according to the above described design procedure are summarized in Table IV. In the last row of the table the measured and theoretical directivity, calculated by means of (10), $D_{m}$, are shown for comparison. Good agreement between the designed, measured and theoretical values was found. The results obtained showed that the proposed design procedure can be used for creation of high efficiency microwave communication antennas. 
TABLE IV. RESULTS OBTAINED FOR THREE BACKFIRE ANTENNAS, $L=2 \Lambda_{0}, 3 \Lambda_{0}$, AND $4 \Lambda_{0}$

\begin{tabular}{|l|c|c|c|}
\hline \multicolumn{1}{|c|}{ Antenna parameter (Dimension) } & Example 1 & Example 2 & Example 3 \\
\hline Design directivity $D$ & $21 \mathrm{~dB}$ & $22.2 \mathrm{~dB}$ & $24 \mathrm{~dB}$ \\
\hline Design frequency $f_{0}$ & $2.2 \mathrm{GHz}$ & $2.25 \mathrm{GHz}$ & $2.2 \mathrm{GHz}$ \\
\hline Approximate length of the antenna $L_{a}$ & $2.10 \lambda_{0}$ & $2.77 \lambda_{0}$ & $4.19 \lambda_{0}$ \\
\hline Final length of the backfire antenna $L$ & $272.7 \mathrm{~mm}\left(2 \lambda_{0}\right)$ & $400.0 \mathrm{~mm}\left(3 \lambda_{0}\right)$ & $545.4 \mathrm{~mm}\left(4 \lambda_{0}\right)$ \\
\hline Optimum phase velocity delay factor $c / v$ & 1.02 & 1.02 & 1.02 \\
\hline Inner diameter $D_{20}$ & $366.4 \mathrm{~mm}\left(2.687 \lambda_{0}\right)$ & $438.8 \mathrm{~mm}\left(3.291 \lambda_{0}\right)$ & $518.2 \mathrm{~mm}\left(3.800 \lambda_{0}\right)$ \\
\hline Diameter of the big reflector $D_{2 C}$ & $559.2 \mathrm{~mm}\left(4.101 \lambda_{0}\right)$ & $669.7 \mathrm{~mm}\left(5.023 \lambda_{0}\right)$ & $790.9 \mathrm{~mm}\left(5.800 \lambda_{0}\right)$ \\
\hline Rim width $w_{1}$ & $34.1 \mathrm{~mm}\left(0.25 \lambda_{0}\right)$ & $33.3 \mathrm{~mm}\left(0.25 \lambda_{0}\right)$ & $34.1 \mathrm{~mm}\left(0.25 \lambda_{0}\right)$ \\
\hline Step width $w_{2}$ & $34.1 \mathrm{~mm}\left(0.25 \lambda_{0}\right)$ & $33.3 \mathrm{~mm}\left(0.25 \lambda_{0}\right)$ & $34.1 \mathrm{~mm}\left(0.25 \lambda_{0}\right)$ \\
\hline Diameter of the small reflector $D_{1}$ & $83.9 \mathrm{~mm}\left(0.615 \lambda_{0}\right)$ & $100.4 \mathrm{~mm}\left(0.753 \lambda_{0}\right)$ & $118.6 \mathrm{~mm}\left(0.870 \lambda_{0}\right)$ \\
\hline Distances $d_{S} d_{R}$ & $34.1 \mathrm{~mm}\left(0.25 \lambda_{0}\right)$ & $33.3 \mathrm{~mm}\left(0.25 \lambda_{0}\right)$ & $34.1 \mathrm{~mm}\left(0.25 \lambda_{0}\right)$ \\
\hline Measured $\left(\right.$ and theoretical) directivity $D_{m}$ & $20.9 \mathrm{~dB}(20.8 \mathrm{~dB})$ & $22.1 \mathrm{~dB}(22.5 \mathrm{~dB})$ & $23.8 \mathrm{~dB}(23.8 \mathrm{~dB})$ \\
\hline
\end{tabular}

\section{ACKNOWLEDGMENT}

The authors wish to acknowledge the financial support under the Chilean Anillos Bicentenario Project ACT-53/2010-2012.

\section{REFERENCES}

[1] H.W. Ehrenspeck, Reflection antenna employing multiple director elements and multiple reflection of energy to effect increased gain, US Patent No. 3,122,745, Febr. 1964, (Filed May 1959).

[2] H.W. Ehrenspeck, "The backfire antenna, a new type of directional line source", Proc. IRE, vol. 48, No. 1, pp. 109$110,1960$.

[3] H.W. Ehrenspeck, “The backfire antenna: new results”. Proc. IEEE, vol. 53, No. 6, pp. 639-641, 1965.

[4] F.J. Zucker, "The backfire antenna: a qualitative approach to its design", Proc. IEEE, vol. 53, No. 7, pp. 746-747, 1965.

[5] J.A. Strom, A dielectric-rod backfire antenna, AFCRL Report 69-0347, August 1969.

[6] H.D. Hristov, Study of backfire antennas with surface-wave structures, Ph.D. Thesis, Sofia, 1973 (In Bulgarian).

[7] A. Kumar, "Theoretical analysis of a long backfire antenna containing a dielectric structure", Microwaves, Opt., and Acoust., vol. 2, No. 3, pp. 91-95, 1978.

[8] G.S. Kirov, H.D. Hristov, "Physical radiation mechanism of a backfire antenna", Nat. Scient. Techn. Conf. "Radar and Radio Relay Lines", Varna, Dig., p.6, Oct. 1980 (In Bulgarian).

[9] H.D. Hristov, Resonator-Type Antennas, D.Sc. Thesis, Sofia, 1987 (In Bulgarian).

[10] A. Kumar, H.D. Hristov, Microwave Cavity Antennas, Artech House, Boston. London, 1989.

[11] W.W. Hansen, J.R. Woodyard, “A new principle in directional antenna design”, Proc. IRE, vol. 26, No. 3, pp.333$345,1938$.

[12] R.C. Jonson, Antenna Engineering Handbook, Third Edition, McGraw-Hill, Inc., 1993.

[13] H.W. Ehrenspeck, H. Poehler, A new method for obtaining maximum gain from Yagi antennas, AFCRC Techn. Rept. No TR-58-355, August 1956.

[14] H. Bach, “Applicability of Hansen-Woodyard condition”, Proc. IEE, vol. 119, No. 1, pp. 38-40, January 1972.

[15] C.A. Balanis, Antenna Theory: Analysis and Design, $3^{\text {rd }}$ edition, Wiley, 2005.

[16] T.A. Milligan, Modern Antenna Design, $2^{\text {nd }}$ edition, John Wiley \& Sons, Inc., 2005.

[17] J.L. Volakis, Antenna Engineering Handbook, McGraw-Hill, 2007.

[18] L. Thourel, Les Antennes, Deuxième edition, Dunod, Paris, 1971. 